\title{
EXPERIMENTAL STUDY OF TREE NETWORKS FOR MINIMAL PUMPING POWER
}

\author{
M.S. SAYEED ${ }^{1}$, I.A. AHMED ${ }^{2}$, A.A. SYED ${ }^{1}$, P.H. RAJU ${ }^{1}$ \& M.S. SALMAN ${ }^{3}$ \\ ${ }^{1}$ Department of Mechanical Engineering, C. Abdul Hakeem College of Engineering and Technology, \\ Melvisharam, Vellore, India. \\ ${ }^{2}$ Department of Electrical Engineering, C. Abdul Hakeem College of Engineering and Technology, \\ Melvisharam, Vellore, India. \\ ${ }^{3}$ Department of Mechanical Engineering, Adhiyamaan Engineering College, Hosur, India.
}

\begin{abstract}
This paper presents an experimental study of constructal tree shaped networks, to optimize the fluid networks based on minimization of pumping power. We take a fresh look at the generation of architectures for fluid flow and instead of minimizing the global flow resistance we focus on minimization of pumping power. Nonsymmetric ( 9 outlets) and dendritic ( 24 outlets) architectures were investigated for both laminar and turbulent flow regimes. A significant reduction in pumping power and volume is observed, compared to conventional flow. These results emphasize the robustness of tree networks for fluid flow.

Keywords: constructal design, Gilbert-Steiner points, pumping power, tree networks.
\end{abstract}

\section{INTRODUCTION}

Recent theoretical developments on constructal theory directs toward predicting macroscopic organization (the occurrence of shape and structure) in natural flow systems, animate and inanimate. The starting point is the question of how to optimize the access between one point and a finite volume (i.e., an infinite number of points). If the volume is an electronic device that generates heat uniformly, access optimization means minimum thermal resistance between the volume and a point-size heat sink. Similarly, if the volume must be bathed at every point by a flow (e.g., air flow in the lung, or blood flow in a capillary bed), optimal access means minimum flow resistance between the volume and a source or sink. The main discovery is purely geometric: any finite-size portion of this composite can have its shape optimized such that its overall resistance to flow is minimal. Consequently, the optimal access solution for the total volume is obtained by optimizing volume shape at every length scale, in a sequence that begins with the smallest building block (elemental system), and proceeds toward larger building blocks (assemblies, constructs). The solution is constructed, hence the "constructal" name of the associated theory. The paths form a tree network in which every single geometric detail is determined theoretically. The tree network cannot be determined theoretically when the time direction is reversed, from large elements toward smaller elements. The constructal principle is further illustrated for fluid flow between a volume and one point, for minimum-time travel between an area and one point and for minimum-cost economic structures [1-6].

Constructal theory proves that the global performance of flow system is maximized. It does not depend on what flows (fluid, heat, electricity, goods, people) but "how" it flows and how it derives its architecture from the competition between objectives and constraints. Constructal theory proclaims the oneness of natural and engineered flow configuration generation phenomena.

A flow system (e.g., tree) has properties that distinguish it from a non-flow system. The properties of the flow system are (1) global external size, e.g., the length scale of the body bathed by the tree flow $L$; (2) global internal size, e.g., the total volume of the ducts $V$; (3) at least one global objective, or performance, e.g., the global flow resistance of the tree $R$; (4) configuration, drawing, architecture; and (5) freedom to morph, i.e., freedom to change the configuration [1].

(C) 2008 WIT Press, www.witpress.com

ISSN: 1755-7437 (paper format), ISSN: 1755-7445 (online), http://journals.witpress.com

DOI: 10.2495/D\&NE-V3-N2-135-149 
Constructal theory strikes the balance between the determinism of chance. In a constructal tree, for example the position of branches can be predicted, but nobody knows exactly how the individual or molecule moves the interstices. Chances and additional constraints will definitely play a role.

\subsection{Objective}

The driving force behind all these developments is the need for "better" performance from our point of view, at our scale, for our benefit. Needed are improvements in the global performance of the macroscopic system. Packing the system with smaller, more powerful and more numerous elemental systems is a necessary first step. The challenge is not only to find geometric arrangements to connect the currents that must access the elemental systems, but to optimize each connection such that, ultimately, each design choice is reflected in an increase in performance at the global level. To construct more and more elements into complex structures and to optimize (with global objective and space constraints) each connection means to construct.

There is continuous striving for the systems to make optimum and so we have designed the architecture from the constructal theory by Bejan and his co-workers. In the first section, we have developed the architecture for conventional flow and compared with non-symmetric tree shaped network $\left(75^{\circ}\right.$ and $\left.56^{\circ}\right)$ for both laminar and turbulent regime. In the second section, we have discussed about the dendritic architecture point-circle flow for both flows for 24 outlets and compared with conventional system.

\section{NON-OPTIMAL NETWORK}

In recent research works on fluid networks, pressure drop has been used extensively as a network of operation cost. Optimal networks were generated by minimizing the pressure drop between the highest and lowest pressures. The very idea of system optimization (in engineering as well as in nature) implies that the system in question is not purposeless: the system has an objective, a duty to fulfill. This task is accomplished at a certain cost, and under global constraints. Identifying these constraints and objectives is the first conceptual step in the process of designing a system. It is a crucial step that calls for adequate modeling. A flawed cost function may lead to "an optimal" design (optimal in the sense that it minimizes the flawed cost function), but there is no guarantee that this design provides a satisfactory performance in view of the "real" cost function. Neither the pressure drop nor the friction resistance are real cost functions, but it is the pumping power.

To begin with, consider the simplest situation: a fully developed laminar flow in a pipe with constant circular cross section. Given the mass flow rate of the pipe, $m$, the pressure drop in the pipe and required pumping power are given by

$$
\begin{aligned}
& \Delta P=\frac{8 \pi v m L}{a^{2}} \\
& W=\frac{8 \pi v m^{2} L}{\rho a^{2}},
\end{aligned}
$$

where $a$ and $L$ are the cross-sectional area and the length of the pipe, respectively. The effect of pipe geometry $(a, L)$ on the pressure drop and pumping power is the same as in the particular case: according to eqns. (1) and (2), $\Delta P$ and $W$ are proportional to $L / a^{2}$. The situation is different when several pipes are connected together to form a network. In that case the total pumping power and 
pressure drop can be written as

$$
\begin{aligned}
& \Delta P=8 \pi v \sum_{e \in \Gamma} \frac{m_{e} L_{e}}{a_{e}^{2}} \\
& W=\frac{8 \pi v}{\rho} \sum_{e} \frac{m_{e}^{2} L_{e}}{a_{e}^{2}} .
\end{aligned}
$$

The summation in eqn. (3) is over the pipes $e$ of a path $\Gamma$ (i.e., over only some of the pipes of the network) from the point of largest pressure to the point of smallest pressure. The summation in eqn. (4) is over all the pipes of the network. It can be shown that the summations in eqns. (3) and (4) are equivalent if: (i) all the pipes of the network (with a given mass flow rate) have the same length and cross-sectional area; (ii) the quantity $m \Omega(m)$ is a constant independent of $m$, where $\Omega(m)$ is the number of pipes with a mass flow rate $m$. When conditions (i) and (ii) described above are not respected, pumping power and pressure drop minimization will lead to different network configurations and levels of performance. Therefore, in general, one cannot deduce that a minimum pressure drop network will look or perform as a minimum pumping power network.

A set of nine outlets and one inlet, as shown in Fig. 1, is connected to the source to transport the fluid. Pressure head is maintained constant for all experiments. As we know, there are two constraints, the first is the total volume of pipe network

$$
V=\sum_{e} a_{e} L_{e} .
$$

The second type of constraint is law of mass conservation at each vertex. The fluid consumption at a given point equals the difference between the inlet and outlet mass flow rates at that point.

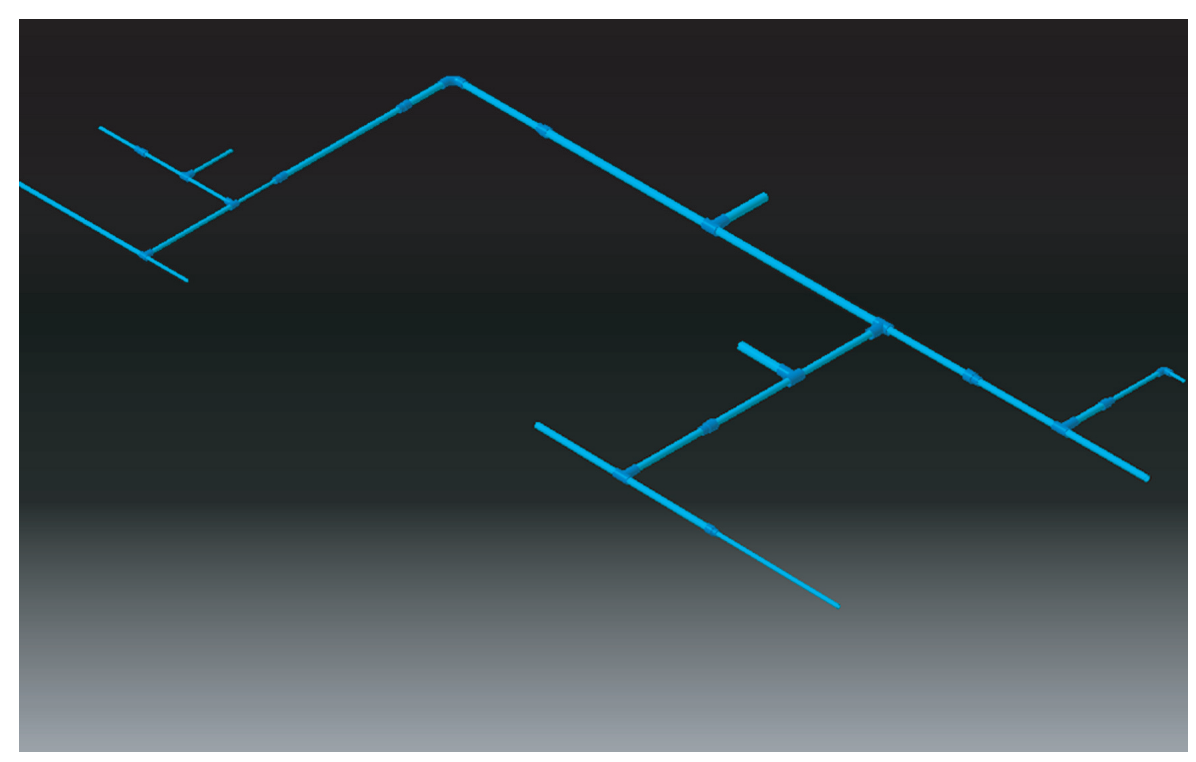

Figure 1: A conventional model for nine outlets. 
There are thus $N$ constraints of this type, one for each vertex

$$
\sum_{j \neq 1} m_{e(i, j)}=s_{i}
$$

The term $s_{i}$ accounts for the fluid consumption of the point $i$. This can be positive or negative depending on whether the vertex $i$ consumes or produces fluid. If $s_{i}=0$, then the vertex $i$ neither uses nor delivers fluid. The optimal network is the one that minimizes the cost function (the pumping power, eqn. (4)) subject to constraints (5) and (6). The non-dimensionalized version of these equations is

$$
\begin{gathered}
W=\sum_{e} \frac{m_{e}^{2} L_{e}}{a_{e}^{2}} \\
V=\sum_{e} a_{e} L_{e}-1=0 \\
M i=\sum_{j \neq 1} m_{e(i, j)}-s_{i}=0 .
\end{gathered}
$$

In summary, the objective is to minimize $W$ in eqn. (7), subject to $N+1$ constraint, eqns. (8) and (9). The parameters that can vary are the cross-sectional area of each pipe, and the mass flow rate that each pipe carries. This means two parameters per pipe. Because there are $N(N+1) / 2$ possible pipes and the $N+1$ constraints, the number of degrees of freedom to minimize pumping power requirement is $\left(N^{2}-2 N-1\right)$.

\subsection{Network for laminar flow}

Natural tree-shaped networks are the best flowing routes between one point and an area (or volume) because they bathe the available space with channels of multiple scales, which are allocated optimally to interstitial areas (or volumes). Many scales are organized hierarchically - few are large and many are small and are distributed non-uniformly, but they are positioned in right places. Trees that do not look like the best perform practically as well as the best.

A key result of constructal theory is flow architecture, optimal spacing, global maximization of flow, overall minimum (fluid and thermal) resistance $[1,2,4,5]$. This becomes an attractive feature of tree-shaped networks in engineering. To understand the properties of tree-shaped flow structures, it is necessary to determine what properties make them better and what strategies designers should employ to arrive at optimal or near-optimal tree constructs faster and more economically.

We conducted experiments for laminar flow with $75^{\circ}$ branches, as shown in Fig. 2, at fixed vertices (i.e., the location of outlets is exactly the same as in a conventional network). A tree network for nine outlets constructed using a $75^{\circ}$ PVC joint is shown in Figs. 3 and 4. The angle obtained is purely empirical as shown in Fig. 2. The angle varies depending upon the flow, as the tree's height is increased the flow regime shifts from turbulent to laminar. This optimized shape not only reduces the discharge time, or in other words increases the mass flow rate for the fixed time, but also minimizes the volume; that is, it reduces $8.5 \%$ of pumping power and $12 \%$ of volume compared to the non-bifurcating model.

\subsection{Network for turbulent flow}

The results presented above for laminar regime can be extended to the turbulent regime. The following empirical relation for the friction factor is commonly used for turbulent fully developed 
flow in a smooth pipe:

$$
f=0.046\left(\frac{2 m}{\mu \pi^{1 / 2} a^{1 / 2}}\right)^{-1 / 5}
$$

Therefore, the dimensionless pumping power required for driving the flow through the pipe $e$ is

$$
W=0.0032 \frac{m_{e}^{14 / 5} L_{e}}{a_{e}^{12 / 5}} .
$$

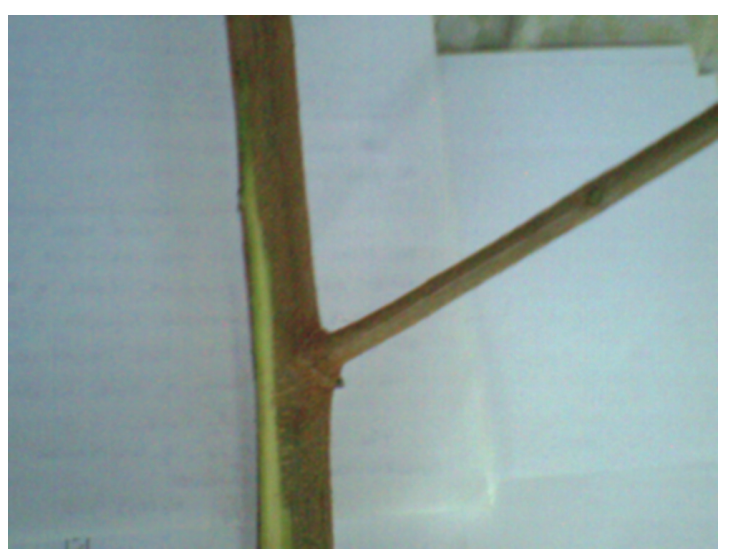

Figure 2: Non-symmetric tree (Royal poinciana) branch which makes an angle of $75^{\circ}$.

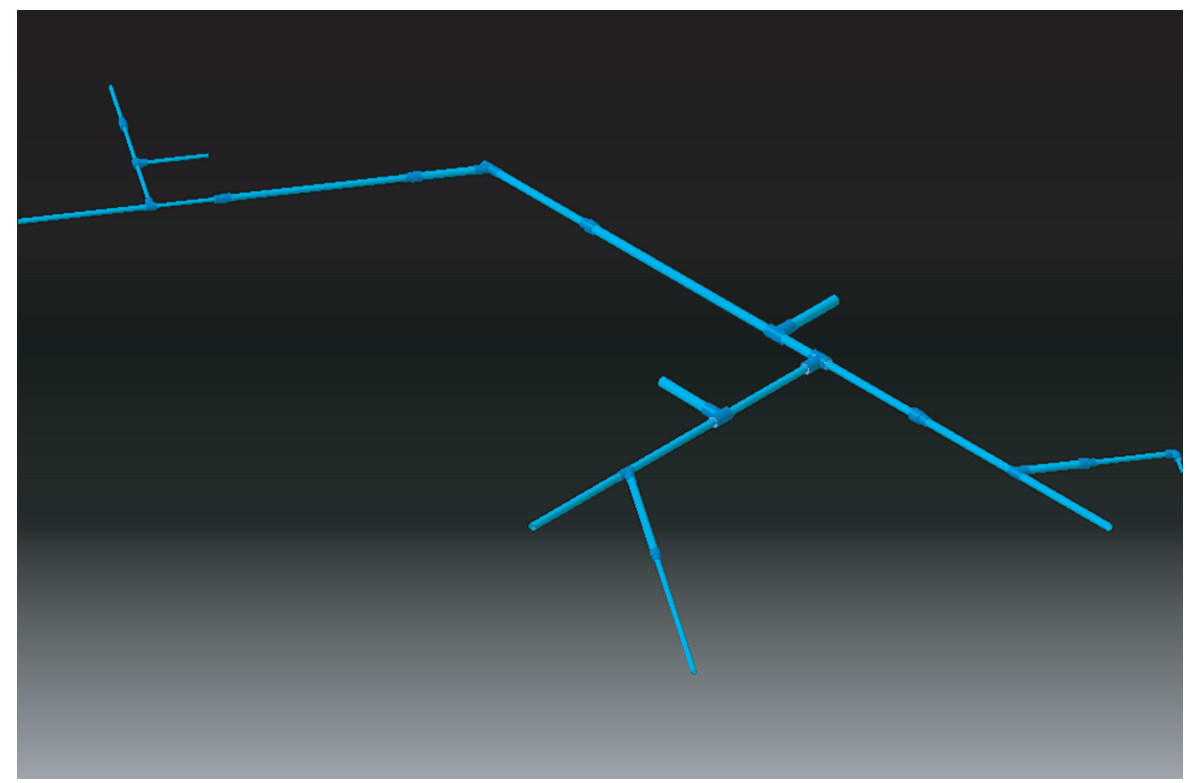

Figure 3: A tree network model for laminar flow with nine outlets. 


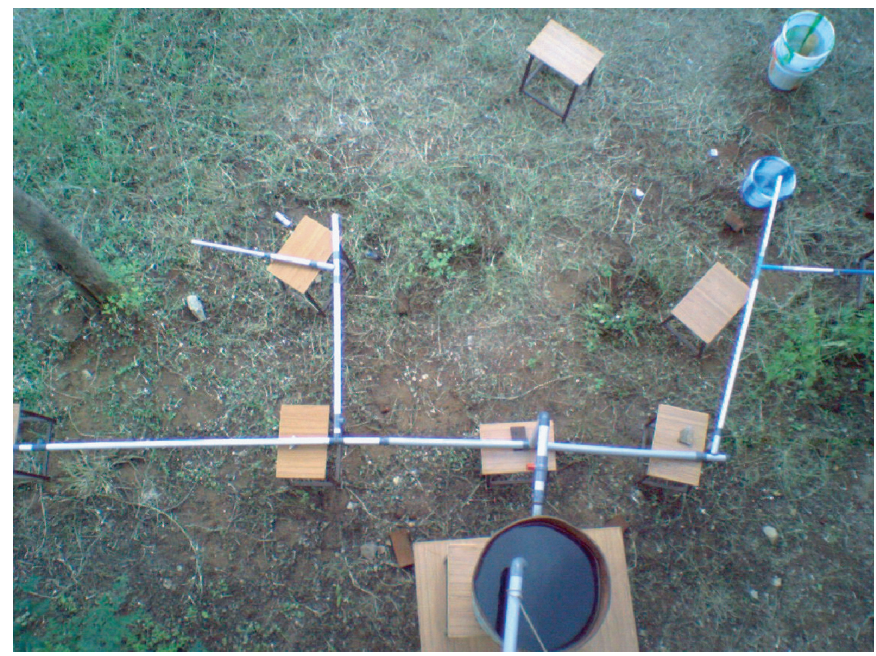

Figure 4: The minimum pumping power tree network $\left(75^{\circ}\right)$ for laminar flow.

Table 1: Values of $\alpha$ and $\varepsilon$ for several flow regimes (reprinted with permission from [11]).

\begin{tabular}{lcc}
\hline Flow regime & $a$ & $\varepsilon$ \\
\hline Fully developed laminar flow & $2 / 3$ & 3 \\
Fully developed turbulent flow in smooth pipes & $14 / 17$ & $17 / 5$ \\
Fully developed turbulent flow in rough pipes & $6 / 7$ & $7 / 2$ \\
\hline
\end{tabular}

The total pumping power for a network where all the pipes are smooth and carry turbulent flow is the sum of the pumping power carried in each pipe. In conclusion, one can state the optimization problem more generally as follows: given set of $N$ points, minimize $\sum_{e} m_{e}^{a} L_{e}$ where $\alpha$ changes when the flow regime changes. The values of $\alpha$ and $\varepsilon$ for different flow regimes are shown in the Table 1. The exponent $\alpha$ is smaller for laminar flow (2/3) and larger for turbulent flow (6/7 for rough pipes; 14/17 for smooth pipes). In all cases, we have $0<\alpha<1$. where $\varepsilon$ also depends upon flow regime: $\varepsilon=3$ for laminar flow, $17 / 3$ for turbulent flow in smooth pipes and $7 / 2$ for turbulent flow in rough pipes. Note that the value of $\varepsilon$ does not influence the optimal configuration of the network. The value of $\alpha$ influences only the relative performance of the network.

The minimum pumping power requirement is

$$
W_{\min } a\left(\sum_{e} m_{e}^{a} L_{e}\right)^{\varepsilon} .
$$

After keen observation of various trees, we have observed that a bifurcation occurs at the base of the trunk, which forms an angle of $56^{\circ}$ (Fig. 5). With the aid of this angle, the optimal network (Fig. 6) is constructed as shown in Fig. 7. Similarly, the results obtained are reduced pumping power of $12 \%$ and volume reduction of $20 \%$ compared to the conventional system. 


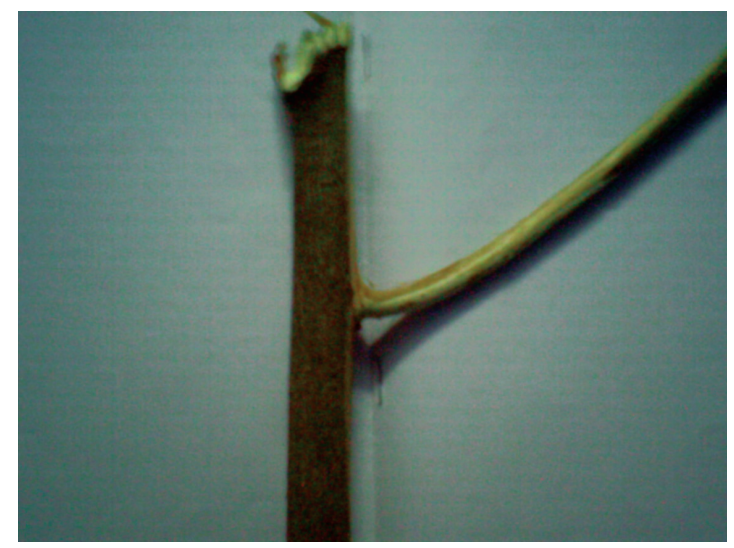

Figure 5: Non-symmetric tree (Royal poinciana) branch which makes an angle of $56^{\circ}$.

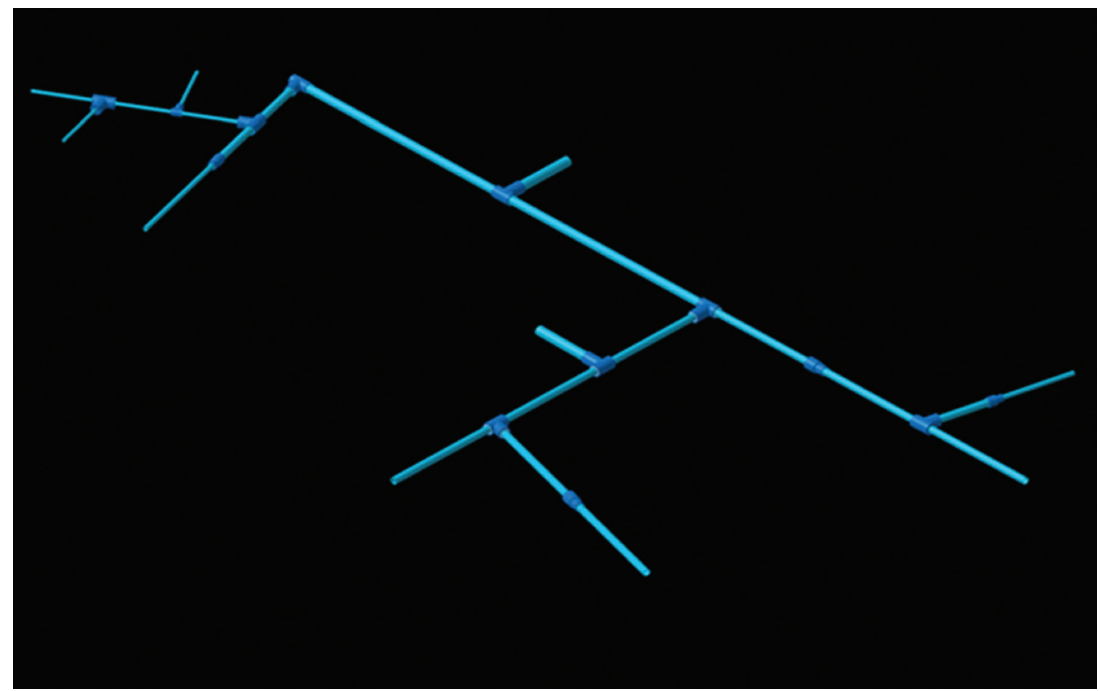

Figure 6: A tree network model for turbulent flow with nine outlets.

A possibility of increase in reduction of pumping power, when the bifurcated diameter is reduced form the main stem diameter. More effort is required to determine the ratio between two diameters of non-symmetric bifurcated Y-shaped structures (in nature).

\section{DENDRITIC FLUID NETWORKS}

The subject of this section is to design a point-volume dendritic network in which new points called Gilbert-Steiner points (GSPs) are added. These points are non-consuming; it greatly increases the number of possible topologies, that is, the number of ways in which one can connect all the points. As the number of consumers $(N)$ increases, the opportunities for adding GSPs also increase.

A new flow structure with minimal resistance is designed, the flow connects the center $f$ disc with the disc perimeter and it proceeds from center to perimeter. The main feature of this structure is 

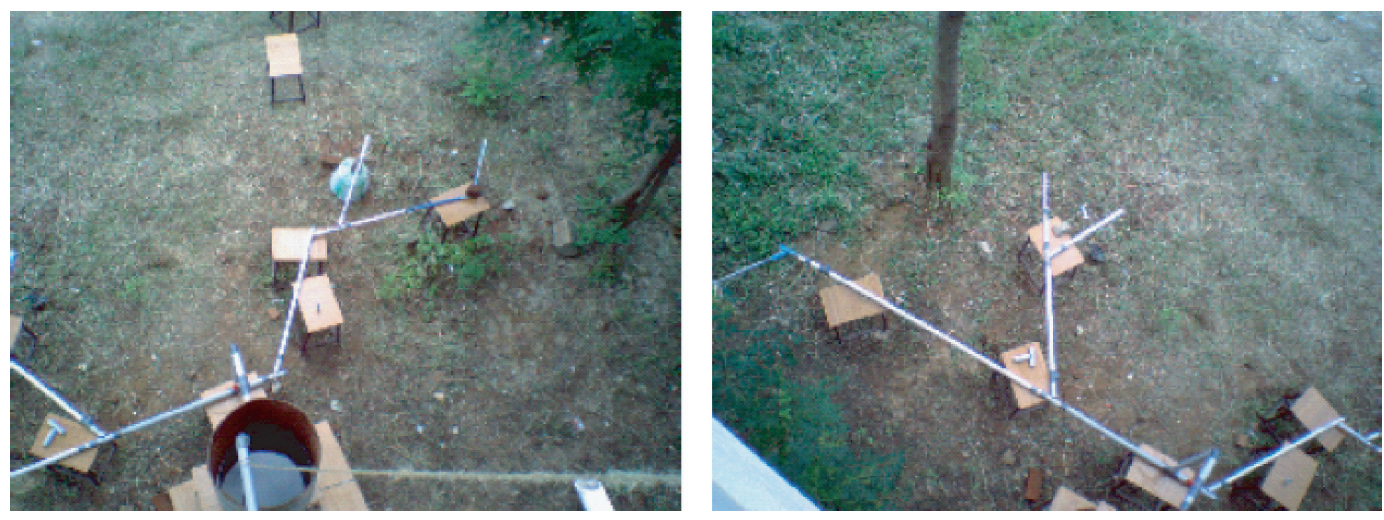

Figure 7: The minimum pumping power tree network for turbulent $\left(56^{\circ}\right)$ flow.

bifurcation at each mode in the network. The ducts are round tubes of several diameters $\left(D_{0}, \ldots, D_{n}\right)$ and lengths $\left(L_{0}, \ldots, L_{n}\right)$. The optimal sizes and lengths of pipes at each pairing level are taken from Murray's study on blood vessels. There is a stepwise change in diameter and length based on this ratio.

$$
\begin{aligned}
\frac{D_{i+1}}{D_{i}} & =2^{-1 / 3} \\
\frac{L_{i+1}}{L_{i}} & =2^{-1 / 3} .
\end{aligned}
$$

The optimized flow architecture has three central tubes $\left(n_{0}=3\right.$; Fig. 8$)$, which are considered to be the best [7, 8]; a similar architecture is observed in a tree with three bifurcations from the center branch as shown in Fig. 9. The pressure drop for three pairing level is proportional to overall flow resistance.

$$
\Delta P=m \frac{8 \pi v}{V^{2}} R^{3} f
$$

where $f$ is the dimensionless flow resistance

$$
f=n_{0}\left(L_{0}+2^{1 / 3} L_{1}+2^{2 / 3} L_{2}+2 L_{3}\right)^{3} .
$$

Bejan et al. prove that the pressure drop factor increases monotonically with $n_{0}$ and indicate that the simplest structure $\left(n_{0}=3\right)$ offers the least global resistance. The adjacent angle between the central tubes is $120^{\circ}$. Figure 9 shows three bifurcations from the stem at an angle of $120^{\circ}$.

To determine the angle between the two adjacent ducts, more elaboration is required. A block as shown in Fig. 8 is transferred into Fig. 10. The degree of an optimal GSP has to be at least three, which means that a GSP must connect at least three pipes. If only one pipe were connected to a GSP, it would be possible to remove the GSP and its corresponding pipe to reduce the pumping power. If a GSP were in contact with only two pipes, then the GSP can be removed with its corresponding pipes, and a new single pipe can be installed between the points that were previously connected to the GSP to reduce the pumping power. Only one of the pipes connected to a GSP can bring fluid to the GSP. In other words, there is only one inflow to an optimal GSP [9]. Otherwise, there would be 


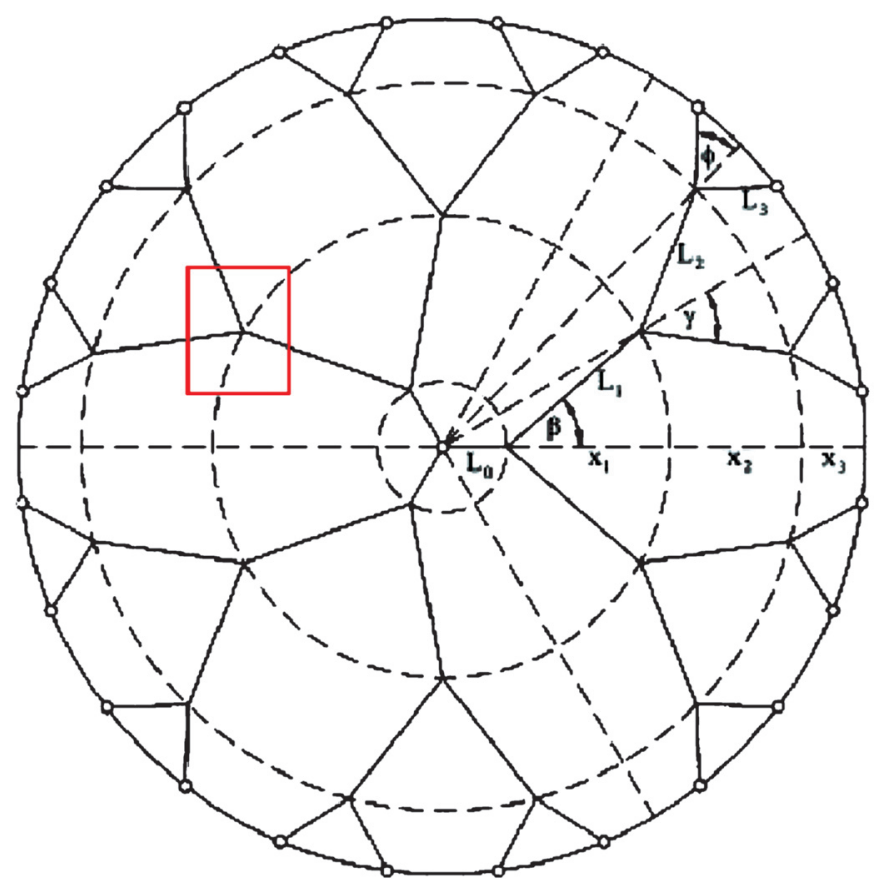

Figure 8: The optimized flow structure with three levels of pairing and $n_{0}=3$ (reprinted with permission from [7]).

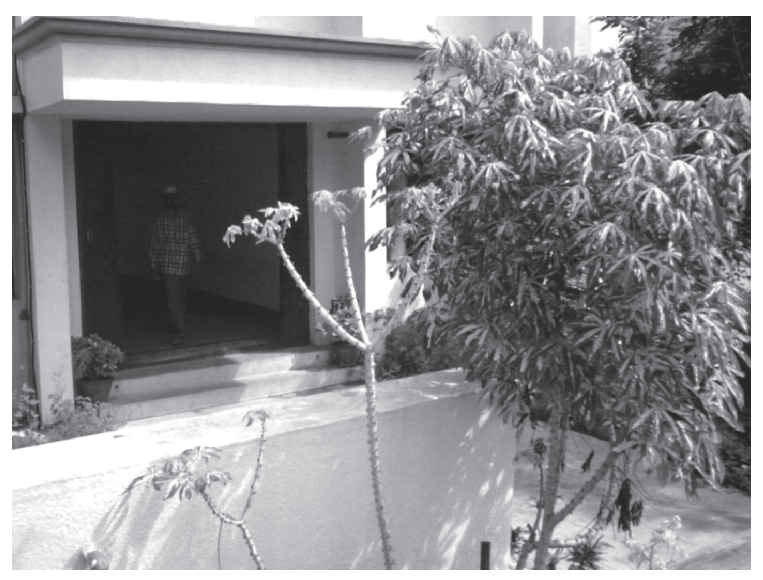

Figure 9: A tree has three bifurcations from the central stem with adjacent angle of $120^{\circ}$.

a loop in the network, the loops are robust in micro scales [10], but it is proven to be inefficient in fluid networks [11].

Next, we examined in greater detail the GSP of degree three: one inflow split into two outflows. Even though this is not the most general case, it is by far the most common GSP encountered in both engineering and nature. Consider Fig. 10. The points $a, b$ and $c$ are given, and the mass flows 
from $a$ to $b$ and $a$ to $c$ are $m_{b}$ and $m_{c}$, respectively. We want to introduce a GSP, named $g$, in order to reduce the cost function. It can be shown that the optimal angles between the pipes are given by eqns. (17-19)

$$
\begin{aligned}
& \cos (b g c)=\frac{\left(m_{b}+m_{c}\right)^{2 a}-\left(m_{b}\right)^{2 a}-\left(m_{c}\right)^{2 a}}{2\left(m_{b}\right)^{a}\left(m_{c}\right)^{a}} \\
& \cos (a g b)=\frac{\left(m_{c}\right)^{2 a}-\left(m_{b}\right)^{2 a}-\left(m_{b}+m_{c}\right)^{2 a}}{2\left(m_{a}+m_{b}\right)^{a}\left(m_{b}\right)^{a}} \\
& \cos (a g c)=\frac{\left(m_{b}\right)^{2 a}-\left(m_{b}+m_{c}\right)^{2 a}-\left(m_{c}\right)^{2 a}}{2\left(m_{b}+m_{c}\right)^{a}\left(m_{c}\right)^{a}},
\end{aligned}
$$

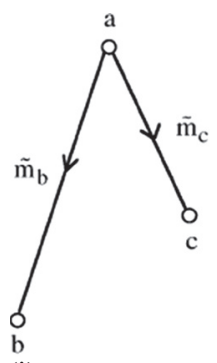

(i)

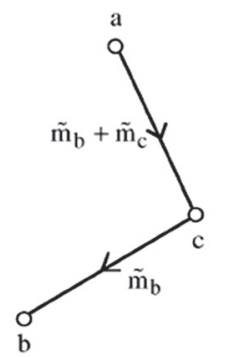

(ii)

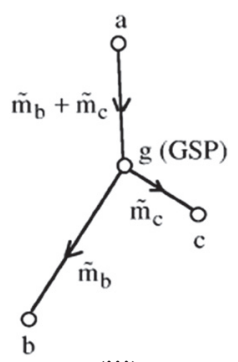

(iii)

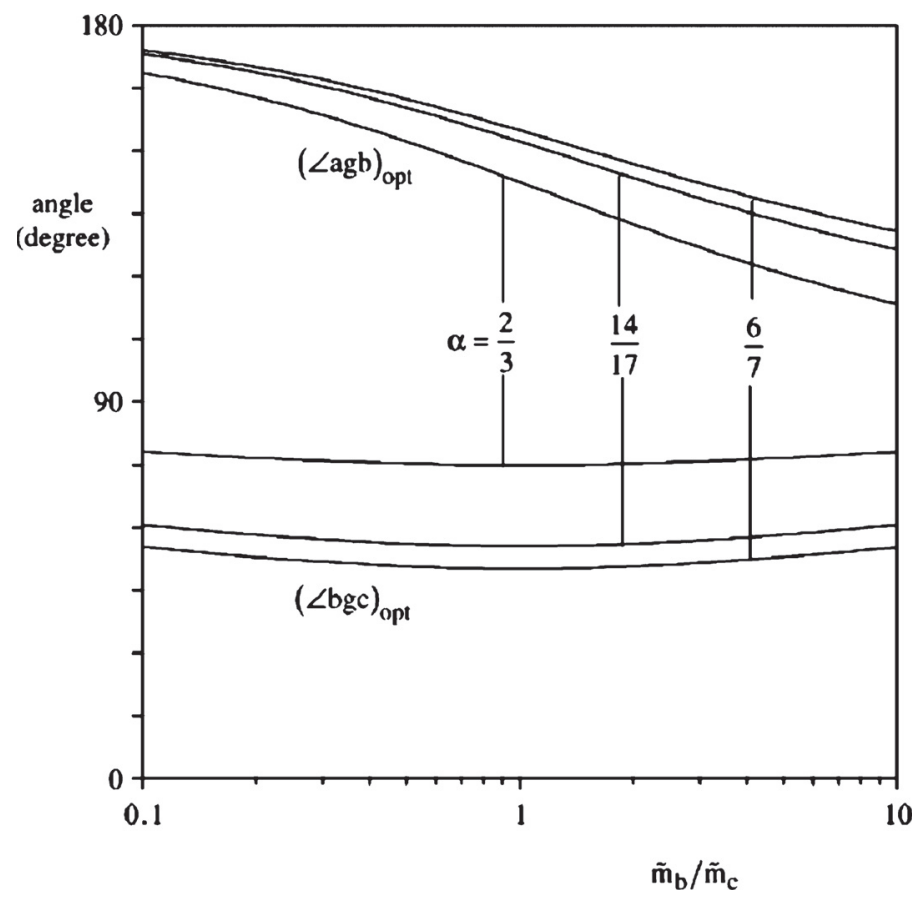

Figure 10: The optimal angles around a GSP of degree three, enqs. (17)-(19) (reprinted with permission from [11]). 
where the parameter $\alpha$ is same as in the Section 2.2 and varies with the flow regime. Figures 11-13 and 14-16 show tree branches that form an angle of $75^{\circ}$ and $56^{\circ}$, respectively, are proved numerically too based on eqns. (17-19).

The angles in eqns. (17)-(19) are reported graphically in Fig. 10. The optimal angle $(b g c)_{\mathrm{opt}}$ is almost constant for all ratios of $m_{b} / m_{c}$, namely $(b g c)_{\text {opt }} \sim 75^{\circ}$ for the laminar regime, $56^{\circ}$ for the turbulent regime, smooth pipes, and $50^{\circ}$ for the turbulent regime, rough pipes.

Experiments are conducted for dendritic architectures for both laminar and turbulent regimes with three pairing levels (i.e., $N=24)$ and with three central tubes $\left(n_{0}=3\right)$. The diameter and lengths are $D_{0}=1.5$ in., $D_{1}=1.25$ in., $D_{2}=1 \mathrm{in} ., D_{3}=0.75$ in and $L_{0}=0.6 \mathrm{~m}, L_{1}=0.476 \mathrm{~m}, L_{2}=0.377 \mathrm{~m}$, $L_{3}=0.3 \mathrm{~m}$, respectively.

A conventional network as shown in Fig. 17 was constructed at fixed vertices with the same 24 outlets. The results are surprising in that a significant reduction in pumping power and volume was observed. The pumping power required for laminar and turbulent flows are $44.5 \%$ and $48.5 \%$

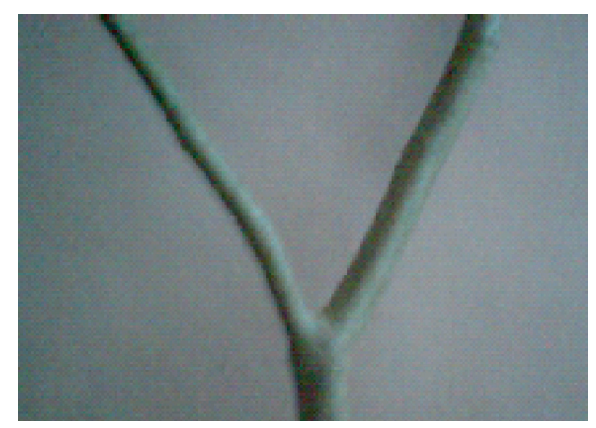

Figure 11: A symmetric tree (Royal poinciana) branch which makes an angle of $75^{\circ}$.

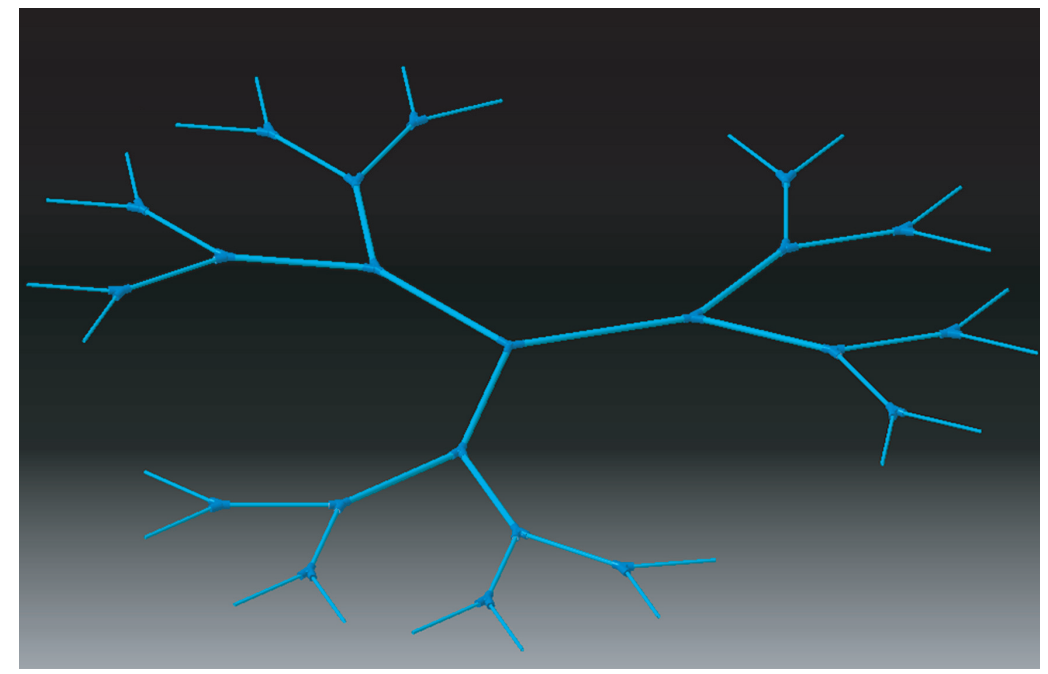

Figure 12: A dendritic model for laminar flow $\left(75^{\circ}\right)$ with three pairing level $(N=24)$ and three central tubes $\left(n_{0}=3\right)$. 


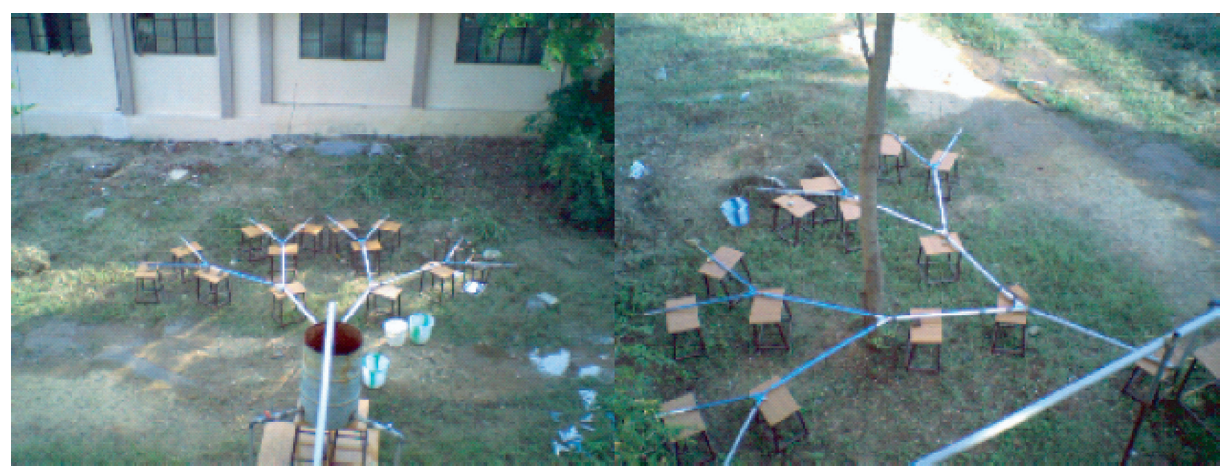

Figure 13: Dendritic architecture for laminar flow $\left(75^{\circ}\right)$ with 24 outlets.

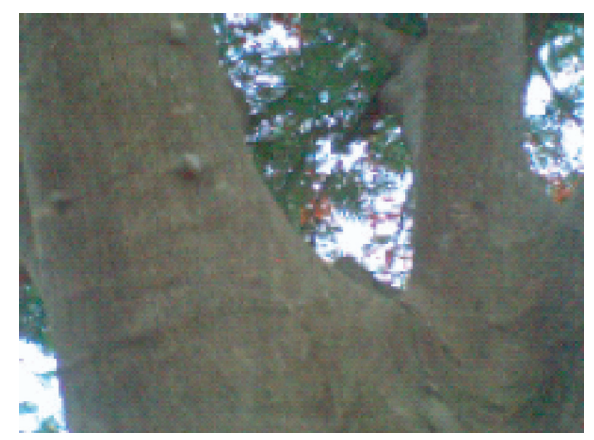

Figure 14: A symmetric tree (Royal poinciana) branch which makes an angle $56^{\circ}$.

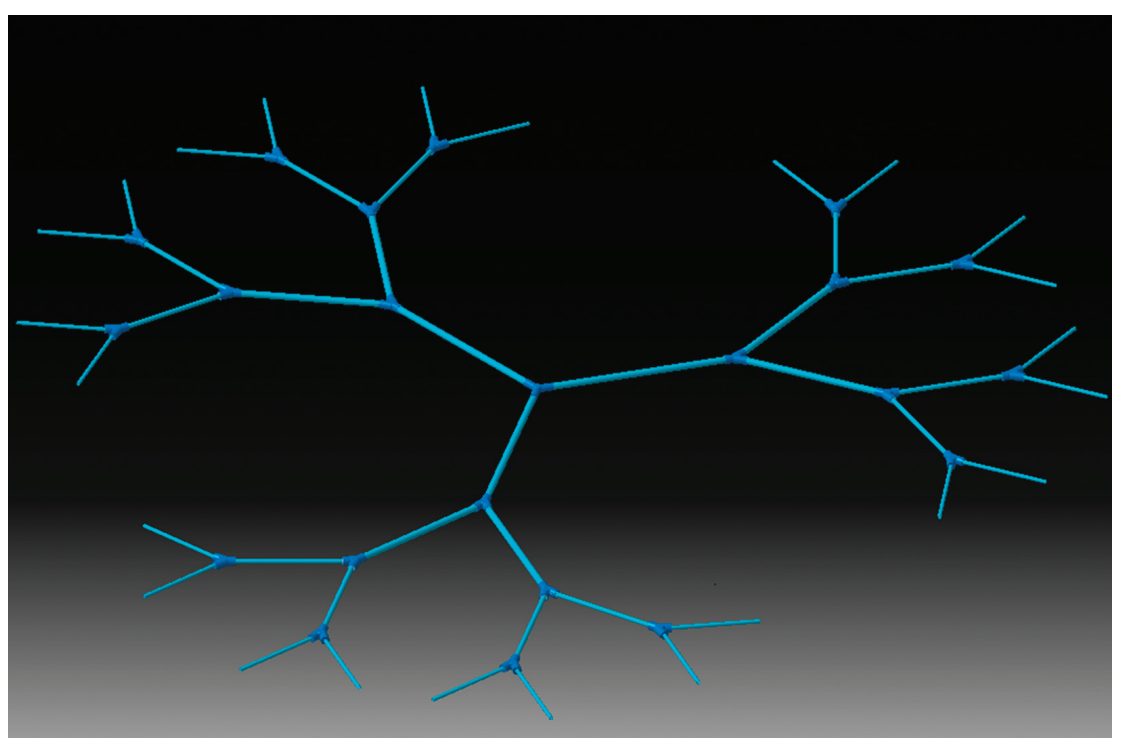

Figure 15: A dendritic model for laminar flow $\left(56^{\circ}\right)$ with three pairing level $(N=24)$ and three central tubes $\left(n_{0}=3\right)$. 


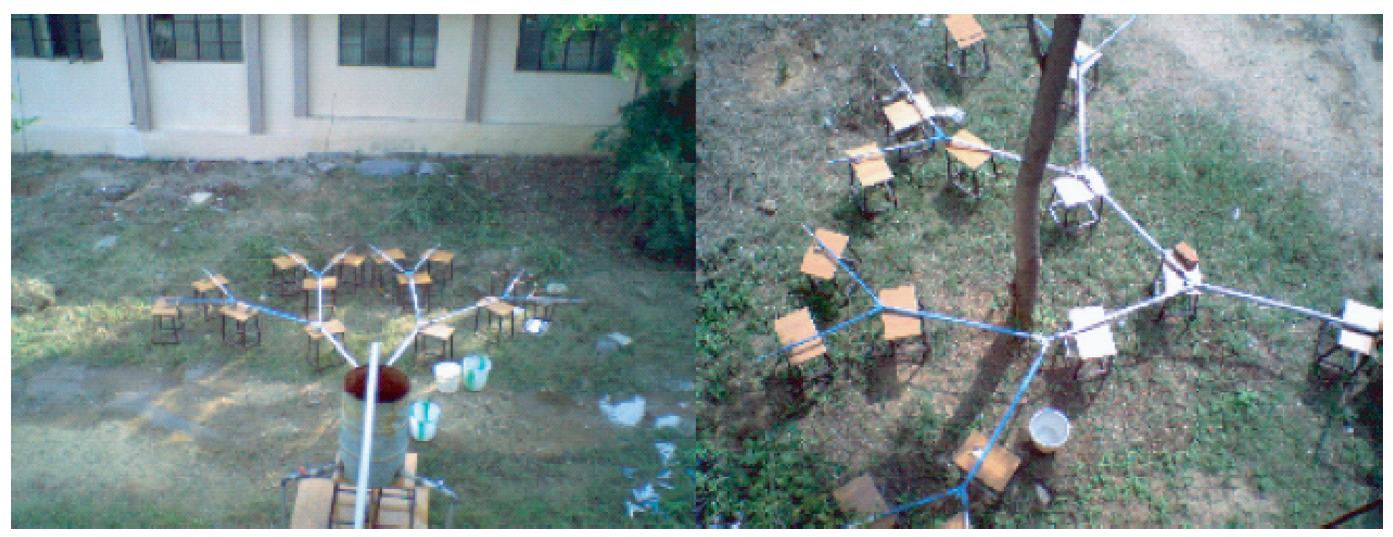

Figure 16: Dendritic architecture for turbulent flow $\left(56^{\circ}\right)$ for 24 outlets.

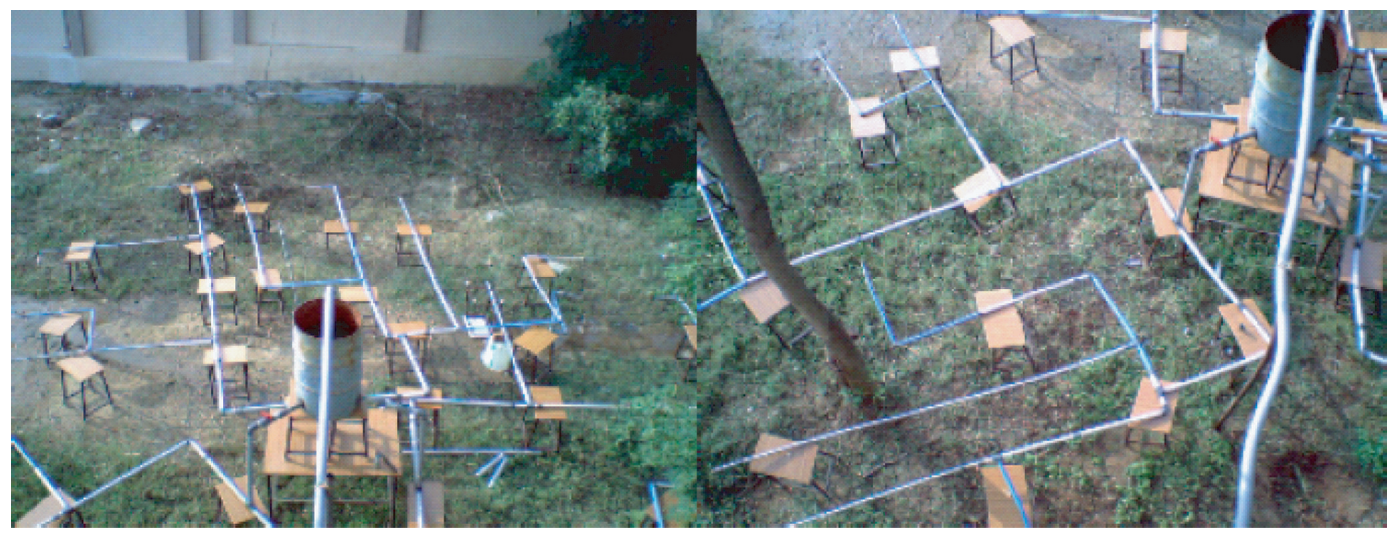

Figure 17: Conventional network with fixed vertices for 24 outlets.

less compared to the conventional system shown in Fig. 17, and the material required is $62 \%$ less compared to the non-optimal network.

\section{CONCLUSION}

Constructal tree networks, introduced by Bejan and his co-workers in recent years, were investigated. It is found that tree networks provide a significant reduction in pumping power and global resistance compared to traditional non-bifurcating systems. The main results presented in this paper can be summarized as follows:

1. With a set of nine vertices for fully developed laminar flow, the network gives the optimal pumping power and volume, reducing the former by $8.5 \%$ and the latter by $12 \%$ compared to a non-bifurcating model.

2. With the same set of nine vertices, which is extended for turbulent flow, the network gives the optimal pumping power and volume, reducing the former by $12 \%$ and the latter by $20 \%$ compared to a conventional system. 
3. Dendritic architectures were investigated for both laminar and turbulent flows based on Murray's optimal diameter and length ratios.

4. Dendritic architectures result in a $44.5 \%$ and $48.5 \%$ reduction of the pumping power for laminar and turbulent flows, respectively, and the total volume of material required is $62 \%$ less compared to a conventional network.

\section{ACKNOWLEDGEMENTS}

We thank Prof. Adrian Bejan for sharing his valuable knowledge and for providing his papers.

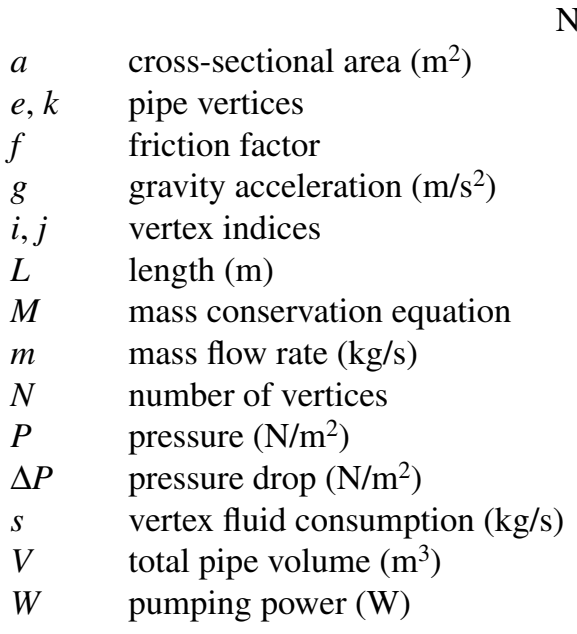

\section{NOMENCLATURE}

Greek symbols

$\alpha, \beta, \gamma$ angles (rad)

$\varepsilon \quad$ exponent to obtain pumping power

$v \quad$ kinematic viscosity $\left(\mathrm{m}^{2} / \mathrm{s}\right)$

$\rho \quad$ density $\left(\mathrm{kg} / \mathrm{m}^{3}\right)$

$\Gamma \quad$ path from larger to lower pressures

\section{REFERENCES}

[1] Bejan, A. \& Lorente, S., Constructal theory of generation of configuration in nature and engineering. Journal of Applied Physics, 100, 041301, August 2006.

[2] Bejan, A., The constructal law of organization in nature: tree shaped flows and body size. Journal of Experimental Biology, 208, pp. 1677-1686, 2005.

[3] Reis, A.H., Constructal theory: from engineering to physics, and how flow systems develop shape and structure. Applied Mechanics Reviews, 59(5), pp. 269-282, 2006.

[4] Bejan, A., Constructal theory: from thermodynamic and geometric optimization to predicting shape in nature. Energy Conservation and Management, 39(16-18), pp. 1705-1718, 1998.

[5] Bejan, A., From heat transfer principles to shape and structure in nature: constructal theory. Journal of Heat Transfer, 122, pp. 430-449, 2000.

[6] Bejan, A. \& Marden, J.H., Unifying constructal theory for scale effects in running, swimming and flying. Journal of Experimental Biology, 209, pp. 238-248, 2006.

[7] Wechsatol, W., Lorente, S. \& Bejan, A., Optimal tree shaped networks for fluid flow in a disc shaped body. International Journal of Heat and Mass transfer, 45(25), pp. 4911-4924, 2002. 
[8] Wechsatol, W., Lorente, S. \& Bejan, A., Dendritic heat convection on a disc. International Journal of Heat and Mass transfer, 46(23), pp. 4381-4391, 2003.

[9] Deo, N., Graph Theory with Applications to Engineering and Computer Science, Prentice Hall: India, 2000.

[10] Wechsatol, W., Lorente, S. \& Bejan, A., Tree networks with loops. International Journal of Heat and Mass Transfer, 48, pp. 573-583, 2005.

[11] Bejan, A. \& Gosselin, L., Tree networks for minimal pumping power. International Journal of Thermal Sciences, 44(1), pp. 53-63, 2005. 